\title{
Dynamics and Matter-Wave Solitons in Bose-Einstein Condensates with Two- and Three-Body Interactions
}

\author{
Jing Chen, ${ }^{1}$ Jie Yang, ${ }^{2}$ and Lu Zhang ${ }^{2}$ \\ ${ }^{1}$ School of Statistics and Mathematics, Central University of Finance and Economics, Beijing 100081, China \\ ${ }^{2}$ School of Science, Beijing Information Science and Technology University, Beijing 100192, China \\ Correspondence should be addressed to Jing Chen; chenjingcufe@163.com
}

Received 8 March 2014; Revised 15 June 2014; Accepted 24 June 2014; Published 5 August 2014

Academic Editor: Victor V. Moshchalkov

Copyright (c) 2014 Jing Chen et al. This is an open access article distributed under the Creative Commons Attribution License, which permits unrestricted use, distribution, and reproduction in any medium, provided the original work is properly cited.

By means of similarity transformation, this paper proposes the matter-wave soliton solutions and dynamics of the variable coefficient cubic-quintic nonlinear Schrödinger equation arising from Bose-Einstein condensates with time-dependent two- and three-body interactions. It is found that, under the effect of time-dependent two- and three-body interaction and harmonic potential with time-dependent frequency, the density of atom condensates will gradually diminish and finally collapse.

\section{Introduction}

Bose-Einstein condensation was first predicted by Einstein and Indian physicist Bose in 1924-1925. It is an exotic quantum phenomenon that was observed in dilute atomic gases for the first time in 1995 [1-3]. The "condensate" here is a state of matter of a dilute gas of bosons at temperatures close to 0 kelvins, which is different from the "condensate" in day life. One of the interesting dynamical features in the context of Bose-Einstein condensate (BEC) is the formation of matter wave solitons such as bright solitons [4, 5], dark solitons [5], vortex solitons [6], and gap solitons [7], which have been experimentally achieved before. Recent experimental techniques for managing nonlinearity have attracted considerable attention. For example, nonlinearity management arises in atomic physics for the Feshbach resonance $[4,8]$ of the scattering length of interatomic interactions in BECs, where the interaction strength can be characterized by a single parameter, the s-wave scattering length $a_{s}$. Across a Feshbach resonance the length $a_{s}$ can in principle be varied from $-\infty$ to $+\infty$, where $a_{s}<0\left(a_{s}>0\right)$ corresponds to effectively attractive (repulsive) interactions. Thus in this situation, one can deal with the governing equations with the nonlinearity coefficients being functions of time or space [4, 9-12].

In the mean-field theory, a BEC system can be well described by the Gross-Pitaevskii (GP) equation [13-15], whose coefficient in front of the cubic term comes from the interatomic interaction. Under certain condition, the GP equation can be converted into the classical nonlinear Schrödinger equation (NLS). It is known that at low densities the three-body interactions can be neglected and the $s$ wave two-body interactions achieve a dominant position. However, the three-body interactions play a key role in BEC at high densities. Similarly, a BEC with two- and three-body interactions can be described by the GP equation with cubicquintic nonlinearity, also called variable coefficient cubicquintic nonlinear Schrödinger (CQNLS) equation [16-18]:

$$
\begin{aligned}
i \frac{\partial \psi}{\partial t} & +\frac{1}{2} \frac{\partial^{2} \psi}{\partial x^{2}}+V(x, t) \psi \\
& +g(t)|\psi|^{2} \psi+G(t)|\psi|^{4} \psi=0,
\end{aligned}
$$

where $\psi$ is matter-wave function, $V(x, t)$ is external potential, $g(t)$ is two-body interaction coefficient, and $G(t)$ is the three-body interaction coefficient. Function $g(t)$ is positive (negative) for attractive (repulsive) condensates and the same as function $G(t)$. Here functions $g(t), G(t)$, and $V(x, t)$ are experimentally controlled.

In this paper, we investigate the matter-wave soliton solutions and dynamics in BEC with two- and three-body interactions trapped by harmonic potential. The paper is organized as follows. In Section 2, the exact matter-wave 
soliton solutions of the variable coefficient cubic-quintic nonlinear Schrödinger equation are obtained by using similarity transformation. In Section 3, the density distributions and dynamics of the matter-wave solitons are investigated by analyzing their figures. We summarize our results in the conclusions.

\section{Exact Matter-Wave Soliton Solutions of CQNLS Equation (1)}

2.1. Similarity Transformation. In what follows, we solve the variable coefficient cubic-quintic nonlinear Schrödinger equation (1) by means of similarity transformation. Through analyzing the properties of the exact solutions, new density distributions and dynamics of BEC with two- and three-body interactions are found.

Assume the variable coefficient CQNLS equation (1) can be transformed into the following CQNLS equation:

$$
i \frac{\partial U}{\partial T}+\frac{\partial^{2} U}{\partial X^{2}}+\sigma_{2}|U|^{2} U+\sigma_{3}|U|^{4} U=0
$$

where $\sigma_{2}, \sigma_{3}$ are constants.

By the principle of similarity transformation, suppose the variable coefficient CQNLS equation (1) has the following form of exact solution:

$$
\begin{gathered}
\psi=\rho U(T, X) e^{i \varphi}, \\
|\psi|^{2}=\rho^{2}|U(T, X)|^{2},
\end{gathered}
$$

where $\rho$ is amplitude, $\varphi$ is phase, and $\rho$ and $\varphi$ are functions $t$ and $x$; function $U(T, X)$ solves the CQNLS equation (2), $T$ is a function of time $t$, and $X$ is a function of $t$ and $x$.

Making use of the symbolic computation software Maple, substituting the similarity transformation (3) and (4) into the variable coefficient CQNLS equation (1), and letting $U(T, X)$ satisfy the CQNLS equation (2), we obtain a set of partial differential equations (PDEs) of functions $T, X, \rho, \varphi, V, g$, and $G$ as

$$
\begin{gathered}
\frac{\partial X}{\partial x}-2 \frac{\partial T}{\partial t}=0 \\
\frac{\partial^{2} X}{\partial x^{2}} \rho+2 \frac{\partial \rho}{\partial x} \frac{\partial X}{\partial x}=0 \\
2 \frac{\partial X}{\partial x} \frac{\partial \varphi}{\partial x} \rho+2 \frac{\partial X}{\partial t} \rho=0 \\
\rho^{2} g-\sigma_{2} \frac{\partial T}{\partial t}=0 \\
2 V \rho-\left(\frac{\partial \varphi}{\partial x}\right)^{2} \rho-2 \frac{\partial \varphi}{\partial t} \rho+\frac{\partial^{2} \rho}{\partial x^{2}}=0 \\
2 \frac{\partial \rho}{\partial t}+\frac{\partial^{2} \varphi}{\partial x^{2}} \rho+2 \frac{\partial \rho}{\partial x} \frac{\partial \varphi}{\partial x}=0 .
\end{gathered}
$$

From the first equation in $(5)$, that is, $(\partial X / \partial x)-2(\partial T / \partial t)=$ 0 , it is found that function $X$ is linear in variable $x$. Furthermore, from $\left(\partial^{2} X / \partial x^{2}\right) \rho+2(\partial \rho / \partial x)(\partial X / \partial x)=0$ we have $(\partial \rho / \partial x)(\partial X / \partial x)=0$. Because $\partial X / \partial x \neq 0$, we have $\partial \rho / \partial x=0$, and then $\rho$ is only a function of time $t$. So we can write functions $X$ and $\rho$ as

$$
\begin{gathered}
X=\tau_{1} x+\tau_{2}, \\
\rho=\rho(t),
\end{gathered}
$$

where $\tau_{1}$ and $\tau_{2}$ are functions of time $t$.

Inserting (6) and (7) into the fourth and fifth equations in (5), we have the expressions of $g$ and $G$ as

$$
g=\frac{\sigma_{2}(\partial T / \partial t)}{\rho^{2}}, \quad G=\frac{\sigma_{3}(\partial T / \partial t)}{\rho^{4}}
$$

Because $\rho$ is only a function of time $t$; from the last equation in (5) we have $2(\partial \rho / \partial t)+\left(\partial^{2} \varphi / \partial x^{2}\right) \rho=0$, so function $\varphi$ is a quadratic function of variable $x$. Thus we can assume function $\varphi$ as

$$
\varphi=\eta_{1} x^{2}+\eta_{2} x+\eta_{3}
$$

where $\eta_{1}, \eta_{2}$, and $\eta_{3}$ are all functions of time $t$.

Finally, the external potential $V(x, t)$ is usually harmonic potential in real experiments, so we let

$$
V(x, t)=\frac{\omega^{2} x^{2}}{2}
$$

where $\omega$ is the frequency of harmonic potential, which is a function of time $t$.

Substituting (6)-(10) into (5) and simplifying, we have

$$
\begin{gathered}
\tau_{1}^{2}-2 \frac{\partial T}{\partial t}=0 \\
2 \tau_{1} \eta_{1} x+\tau_{1} \eta_{2}+\frac{\partial \tau_{1}}{\partial t} x+\frac{\partial \tau_{2}}{\partial t} x=0 \\
4 \eta_{1}^{2} x^{2}+4 \eta_{1} \eta_{2} x+\eta_{2}^{2} \\
+2 \frac{\partial \eta_{1}}{\partial t} x^{2}+2 \frac{\partial \eta_{2}}{\partial t} x+2 \frac{\partial \eta_{3}}{\partial t}-\omega^{2} x^{2}=0 \\
\frac{\partial \rho}{\partial t}+\eta_{1} \rho=0
\end{gathered}
$$

which become the following ordinary differential equations (ODEs) of functions $\tau_{1}, \tau_{2}, \eta_{1}, \eta_{2}, \eta_{2}, T$, and $\rho$ by further calculating

$$
\begin{gathered}
\tau_{1}^{2}-2 \frac{\partial T}{\partial t}=0, \\
\tau_{1} \eta_{2}+\frac{\partial \tau_{2}}{\partial t}=0, \quad 2 \tau_{1} \eta_{1}+\frac{\partial \tau_{1}}{\partial t}=0, \\
2 \frac{\partial \eta_{2}}{\partial t}+4 \eta_{1} \eta_{2}=0, \quad \omega^{2}-2 \frac{\partial \eta_{1}}{\partial t}-4 \eta_{1}^{2}=0, \\
2 \frac{\partial \rho}{\partial t}+2 \eta_{1} \rho=0, \quad-2 \frac{\partial \eta_{3}}{\partial t}-\eta_{2}{ }^{2}=0 .
\end{gathered}
$$


The exact solutions of the ODEs in (15)-(18) are

$$
\begin{gathered}
\eta_{1}=\eta_{1}, \\
\tau_{1}=C_{6} e^{-2 \int \eta_{1} d t}, \\
\eta_{2}=C_{5} e^{-2 \int \eta_{1} d t}, \\
T=\frac{1}{2} \int \tau_{1}^{2} d t+C_{4}, \\
\eta_{3}=-\frac{1}{2} \int \eta_{2}^{2} d t+C_{3}, \\
\rho=C_{2} e^{-\int \eta_{1} d t}, \\
\omega= \pm \sqrt{2\left(\frac{\partial \eta_{1}}{\partial t}+2 \eta_{1}^{2}\right),} \\
\tau_{2}=-\int \tau_{1} \eta_{2} d t+C_{1},
\end{gathered}
$$

where we assume the frequency of the harmonic potential to be positive; that is, $\omega=\sqrt{2\left(\left(\partial \eta_{1} / \partial t\right)+2 \eta_{1}^{2}\right)}$, and $C_{1}, \ldots, C_{6}$ are constants.

Now we discuss the frequency of the harmonic potential $\omega$ by six cases.

Case 1. Let $\omega=\Omega_{0}$ (constant); that is, the frequency of the harmonic potential is invariant. Then we can get function $\eta_{1}$ from $\omega=\sqrt{2\left(\left(\partial \eta_{1} / \partial t\right)+2 \eta_{1}^{2}\right)}$. So other variables can be determined by (19)-(26). In current case, the function $\eta_{1}$ is

$$
\eta_{1}=-\frac{\Omega_{0}}{2} \frac{e^{2 \Omega_{0} t}+e^{2 C_{0} \Omega_{0}}}{e^{2 C_{0} \Omega_{0}}-e^{2 \Omega_{0} t}}
$$

where $\Omega_{0}$ is a constant.

Case 2. Let function $\eta_{1}$ be linear in time $t$; that is, $\eta_{1}=\Omega_{1} t$ with $\Omega_{1}$ a constant, and then we have

$$
\omega=\sqrt{2 \Omega_{1}\left(1+2 \Omega_{1} t^{2}\right)} .
$$

Case 3. Let function $\eta_{1}$ be an exponential function of time $t$; that is, $\eta_{1}=e^{\Omega_{2} t}$, and then we have

$$
\omega=\sqrt{2 e^{t \Omega_{2}}\left(2 e^{t \Omega_{2}}+\Omega_{2}\right)} .
$$

Case 4. Let function $\eta_{1}$ be a hyperbolic function of time $t$; that is, $\eta_{1}=\cosh \left(\Omega_{3} t\right)$, and then we have

$$
\omega=\sqrt{2 \sinh \left(\Omega_{3} t\right)+4 \cosh \left(\Omega_{3} t\right)^{2}},
$$

where sinh denotes hyperbolic sine function and cosh denotes hyperbolic cosine function.
Case 5. Let function $\eta_{1}$ be another exponential function of time $t$; that is, $\eta_{1}=e^{\Omega_{4} t^{2}}$, and then we have

$$
\omega=2 \sqrt{e^{\Omega_{4} t^{2}}\left(e^{\Omega_{4} t^{2}}+\Omega_{4} t\right)} .
$$

Case 6. Finally, let function $\eta_{1}$ be $\eta_{1}=e^{\sin \left(\Omega_{5} t\right)}$, and then we have

$$
\omega=\sqrt{2 e^{\sin \left(\Omega_{5} t\right)}\left[2 e^{\sin \left(\Omega_{5} t\right)}+\Omega_{5} \cos \left(\Omega_{5} t\right)\right]},
$$

and here and above $\Omega_{1}, \Omega_{2}, \ldots, \Omega_{5}$ are nonzero constants.

2.2. Exact Solutions of the CQNLS Equation (2). Up to now, we have derived the coefficients in the similarity transformation. The next thing is to find the exact solutions of the CQNLS equation (2) to formulate the wave function $U(T, X)$. In what follows, we list three types of exact solutions of the CQNLS equation (2).

Type 1. Consider

$$
U_{1}(T, X)=\frac{c n\left(l_{1} X+l_{2} T, k\right) e^{i\left(l_{0} X+d_{0} T\right)}}{\sqrt{d_{1}+d_{2}\left[c n\left(l_{1} X+l_{2} T, k\right)\right]^{2}}},
$$

where $c n$ is Jacobi elliptic cosine function [19, 20] with module $k(0 \leq k \leq 1)$, and $l_{0}$ and $d_{2}$ are nonzero constants and

$$
\begin{gathered}
d_{1}=-\frac{\left(4 K^{2} k^{2}+\sigma_{2} d_{2}+2 \sigma_{3}-2 K^{2}\right) d_{2}}{4 K^{2} k^{2}} \\
l_{1}=\frac{K}{d_{2}}, \quad l_{2}=-2 \frac{K l_{0}}{d_{2}}, \\
d_{0}=\frac{-4 l_{0}^{2} d_{2}^{2}+3 \sigma_{2} d_{2}-4 K^{2} k^{2}+2 \sigma_{3}+2 K^{2}}{4 d_{2}^{2}}
\end{gathered}
$$

where the constant $K$ satisfies

$$
\begin{aligned}
K=\frac{1}{6}(- & 24 \sigma_{3} k^{2}+12 \sigma_{3} \\
& \left.+6 \sqrt{9 \sigma_{2}^{2} d_{2}^{2}+24 \sigma_{2} \sigma_{3} d_{2}+\left(16 k^{4}-16 k^{2}+16\right) \sigma_{3}^{2}}\right)^{1 / 2} .
\end{aligned}
$$


Type 2. Consider

$$
U_{2}(T, X)=\frac{\sqrt{2} \operatorname{sech} \sqrt{6 Q-12 \sigma_{3}}\left(X-2 l_{0} T\right) e^{i\left(\left(12 l_{0} d_{2}{ }^{2} X+\left(8 \sigma_{3}-12 l_{0}{ }^{2} d_{2}{ }^{2}+9 \sigma_{2} d-Q\right) T\right) / 12 d_{2}{ }^{2}\right)}}{\sqrt{d_{2}\left[3 \sigma_{2} d_{2}+4 \sigma_{3}+Q+2 \operatorname{sech}^{2}\left(\left(\sqrt{6 Q-12 \sigma_{3}}\left(X-2 l_{0} T\right)\right) / 6 d_{2}\right)\left(2 \sigma_{3}-Q\right)\right]\left(2 \sigma_{3}-Q\right)^{-1}}}
$$

where $l_{0}$ and $d_{2}$ are nonzero constants and $Q=$ $\sqrt{\left(4 \sigma_{3}+3 \sigma_{2} d_{2}\right)^{2}}$.

Type 3. Consider

$$
U_{3}(T, X)=\frac{\sinh \left(\left(\sqrt{-2 \sigma_{2} d_{2}-4 \sigma_{3}} / 2 d_{2}\right)\left(X-2 l_{0} T\right)\right) e^{i\left(\left(l_{0} X d_{2}{ }^{2}+\left(\sigma_{2} d_{2}+\sigma_{3}+l_{0}{ }^{2} d_{2}{ }^{2}\right) T\right) / d_{2}{ }^{2}\right)}}{\sqrt{d_{2}\left[3 \sigma_{2} d_{2}+6 \sigma_{3}+\sinh ^{2}\left(\left(\sqrt{-2 \sigma_{2} d_{2}-4 \sigma_{3}}\left(X-2 l_{0} T\right)\right) / 2 d_{2}\right)\left(4 \sigma_{3}+3 \sigma_{2} d_{2}\right)\right]\left(4 \sigma_{3}+3 \sigma_{2} d_{2}\right)^{-1}}}
$$

where $l_{0}$ and $d_{2}$ are nonzero constants.

2.3. Exact Matter-Wave Soliton Solutions of (1). Three types of exact solutions $U_{1}(T, X), U_{2}(T, X), U_{3}(T, X)$ of the CQNLS equation (2) have been obtained above. In order to achieve the exact matter-wave soliton solutions of the variable coefficient CQNLS equation (1), we only need to combine the solutions $U_{1}(T, X), U_{2}(T, X), U_{3}(T, X)$ with the similarity transformation (3). Thus the exact matter-wave soliton solution of the variable coefficient CQNLS equation is

$$
\psi(x, t)=\rho U(T, X) e^{i \varphi},
$$

where X, $\varphi$, and $\rho$ are given by (6), (9), and (24) and functions $\tau_{1}, \tau_{2}, \eta_{1}, \eta_{2}, \eta_{3}$, and $T$ are given by (19)-(26). Here the value of function $\eta_{1}$ is the key to the solution. We have listed six cases of choices of function $\eta_{1}$ above. But we only choose $\eta_{1}=\Omega_{1} t$ in the following calculation. So from (19)-(26), we have

$$
\begin{gathered}
\omega=\sqrt{2 \Omega_{1}\left(1+2 \Omega_{1} t^{2}\right),} \\
\eta_{2}=C_{5} e^{-\Omega_{1}{ }^{2} t^{2}}, \\
\eta_{3}=-\frac{C_{5} C_{6}}{2} \frac{\sqrt{2 \pi} \operatorname{erf}\left(\sqrt{2} \Omega_{1} t\right)}{4 \Omega_{1}}, \\
T=\frac{C_{6}{ }^{2}}{2} \frac{\sqrt{2 \pi} \operatorname{erf}\left(\sqrt{2} \Omega_{1} t\right)}{4 \Omega_{1}}+C_{4},
\end{gathered}
$$$$
\psi_{2}(t, x)=C_{2} e^{-\Omega_{1} t^{2} / 2}
$$

$$
\begin{aligned}
& \cdot \frac{\sqrt{2} \operatorname{sech} \sqrt{6 Q-12 \sigma_{3}}\left(X-2 l_{0} T\right) e^{i\left(\left(12 l_{0} X d_{2}{ }^{2}\left(8 \sigma_{3}-12 l_{0}{ }^{2}+9 \sigma_{2} d_{2}-Q\right) T\right) / 12 d_{2}{ }^{2}\right)}}{\sqrt{d_{2}\left[3 \sigma_{2} d_{2}+4 \sigma_{3}+Q+2 \operatorname{sech}^{2}\left(\left(\sqrt{6 Q-12 \sigma_{3}}\left(X-2 l_{0} T\right)\right) / 6 d_{2}\right)\left(2 \sigma_{3}-Q\right)\right]\left(2 \sigma_{3}-Q\right)^{-1}}} \\
& \cdot e^{i\left[\Omega_{1} t x^{2}+C_{5} e^{-\Omega_{1}{ }^{2} t^{2}} x-\left(C_{5} C_{6} / 2\right)\left(\sqrt{2 \pi} \operatorname{erf}\left(\sqrt{2} \Omega_{1} t\right) / 4 \Omega_{1}\right)\right]} \cdot
\end{aligned}
$$

where erf is error function (also named Gaussian error function). harmonic potential $V(x, t)=2 \Omega_{1}\left(1+2 \Omega_{1} t^{2}\right) x^{2}$, the coefficients of two- and three-body interactions are $g(t)=$ $\left(\sigma_{2} C_{6}{ }^{2} / 2 \rho^{2}\right) e^{-2 \Omega_{1}{ }^{2} t^{2}}, G(t)=\left(\sigma_{3} C_{6}{ }^{2} / 2 \rho^{4}\right) e^{-2 \Omega_{1}{ }^{2} t^{2}}$, and we derive three families of exact matter-wave soliton solutions of the variable coefficient CQNLS equation (1) as follows.

Family 1. Consider

$$
\begin{aligned}
\psi_{1}(t, x)= & C_{2} e^{-\Omega_{1} t^{2} / 2} \\
& \cdot \frac{c n\left(l_{1} X+l_{2} T, k\right) e^{i\left(l_{0} X+d_{0} T\right)}}{\sqrt{d_{1}+d_{2}\left[c n\left(l_{1} X+l_{2} T, k\right)\right]^{2}}} \\
& \cdot e^{i\left[\Omega_{1} t x^{2}+C_{5} e^{-\Omega_{1}{ }^{2} t^{2}} x-\left(C_{5} C_{6} / 2\right)\left(\left(\sqrt{2 \pi} \operatorname{erf}\left(\sqrt{2} \Omega_{1} t\right)\right) / 4 \Omega_{1}\right)\right]} .
\end{aligned}
$$

Family 2. Consider

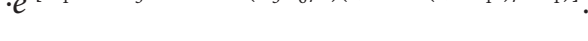

To sum up, when the external potential is time-dependent 
Family 3. Consider

$$
\begin{aligned}
& \psi_{3}(t, x)=C_{2} e^{-\Omega_{1} t^{2} / 2} \\
& \sinh \left(\left(\sqrt{-2 \sigma_{2} d_{2}-4 \sigma_{3}} / 2 d_{2}\right)\left(X-2 l_{0} T\right)\right) e^{i\left(\left(l_{0} X d_{2}{ }^{2}+\left(\sigma_{2} d_{2}+\sigma_{3} l_{0}{ }^{2} d_{2}{ }^{2}\right) T\right) / d_{2}{ }^{2}\right)} \\
& \sqrt{d_{2}\left[3 \sigma_{2} d_{2}+6 \sigma_{3}+\sinh ^{2}\left(\left(\sqrt{-2 \sigma_{2} d_{2}-4 \sigma_{3}} / 2 d_{2}\right)\left(X-2 l_{0} T\right)\right)\left(4 \sigma_{3}+3 \sigma_{2} d_{2}\right)\right]\left(4 \sigma_{3}+3 \sigma_{2} d_{2}\right)^{-1}} \\
& \cdot e^{i\left[\Omega_{1} t x^{2}+C_{5} e^{-\Omega_{1}^{2} t^{2}} x-\left(C_{5} C_{6} / 2\right)\left(\sqrt{2 \pi} \operatorname{erf}\left(\sqrt{2} \Omega_{1} t\right) / 4 \Omega_{1}\right)\right]},
\end{aligned}
$$

where the functions $T, X$ are given by (39) and $Q=$ $\sqrt{\left(4 \sigma_{3}+3 \sigma_{2} d_{2}\right)^{2}}$.

Remark 1. By considering the rest five cases of frequency $\omega$ and function $\eta_{1}$, we can also obtain other exact matter-wave soliton solutions of the variable coefficient CQNLS equation (1) under different types of two- and three-body interactions.

\section{Density Distributions and Dynamics of the Matter-Wave Solitons}

In this Section, we investigate the density distributions and dynamics of the matter-wave soliton solutions (40)-(42) by analyzing their figures. It is noted that the frequency of the harmonic potential is $\omega=\sqrt{2 \Omega_{1}\left(1+2 \Omega_{1} t^{2}\right)}$, and the coefficients of two- and three-body interactions are $g(t)=$ $\left(\sigma_{2} C_{6}{ }^{2} / 2 \rho^{2}\right) e^{-2 \Omega_{1}{ }^{2} t^{2}}, G(t)=\left(\sigma_{3} C_{6}{ }^{2} / 2 \rho^{4}\right) e^{-2 \Omega_{1}{ }^{2} t^{2}}$, which are monotone nonincreasing function of time $t$.

3.1. Density Distribution and Dynamics of Solution $\psi_{1}(x, t)$. For the matter-wave soliton solution $\psi_{1}(x, t)$ in $(40)$, we choose the parameters as follows: $\mathrm{C}_{1}=\mathrm{C}_{2}=\cdots=\mathrm{C}_{6}=1$, $\Omega_{1}=0.01, \sigma_{2}=-0.5, \sigma_{3}=-1, d_{2}=2, l_{0}=1$, and $k=0.99$. Here the time range is $[0,15]$, time size is 0.01 , the space range is $[-20,20]$, and space size is 0.05 .

The density distributions of the matter-wave soliton solution $\psi_{1}(x, t)$ are shown in Figure 1. Here the coefficient of two-body interaction is $g(t)<0$ and that of threebody interaction is $G(t)<0$, which denotes that both twobody and three-body interactions are repulsive. According to the periodical property of the Jacobi elliptic functions, the density distribution $\left|\psi_{1}(x, t)\right|^{2}$ is periodic. We can choose elliptic modulus $k$ from $0<k<1$, and we let $k=0.99$ here. It is observed from Figure 1 that there are five peaks in the density distribution; that is, it has five periods in space. Under the effects of time-dependent harmonic potential and the repulsive two- and three-body interactions, the density distribution of wave function $\psi_{1}(x, t)$ decreases with time. We can also get various density distributions by choosing $k$ from $0<k<1$.

3.2. Density Distribution and Dynamics of Solution $\psi_{2}(x, t)$. We now study the matter-wave soliton solution $\psi_{2}(x, t)$ in (41). The parameters here are $C_{1}=C_{2}=\cdots=C_{6}=1$,
$\Omega_{1}=0.01, \sigma_{2}=1 / 3, \sigma_{3}=-0.5, d_{2}=-1$, and $l_{0}=1$. The time range is $[0,10]$, time size is 0.05 , the space range is $[-200,200]$, and space size is 0.1 .

The density distributions of the matter-wave soliton solution $\psi_{2}(x, t)$ are shown in Figure 2. Here the coefficient of two-body interaction is $g(t)>0$, and that of threebody interaction is $G(t)<0$, which denotes that the twobody interaction is attractive and the three-body interaction is repulsive. This is a matter-wave bright soliton and is a localized nonlinear wave. It is seen from the color bar that as time goes on the density distribution of wave function, $\psi_{2}(x, t)$ diminishes. Thus we find that attractive two-body interaction and repulsive three-body interaction do not support stable matter-wave bright soliton, which is consistent with the real experiments.

3.3. Density Distribution and Dynamics of Solution $\psi_{3}(x, t)$. Finally, we analyze the matter-wave soliton solution $\psi_{3}(x, t)$ in (42). The parameters here are $\mathrm{C}_{1}=\mathrm{C}_{2}=\cdots=\mathrm{C}_{6}=1$, $\Omega_{1}=0.01, \sigma_{2}=-2, \sigma_{3}=-1, d_{2}=5$, and $l_{0}=1$. The time range is $[0,10]$, time size is 0.02 , the space range is $[-10,10]$, and space size is 0.01 .

The density distributions of the matter-wave soliton solution $\psi_{3}(x, t)$ are shown in Figure 3. Here the coefficient of two-body interaction is $g(t)<0$, and that of three-body interaction is $G(t)<0$, which denotes that both two-body interaction and three-body interactions are repulsive. This is a matter-wave dark soliton and is also a localized nonlinear wave. It is also observed that the density distribution of wave function $\psi_{3}(x, t)$ also diminishes with time.

\section{Conclusions}

In summary, we have studied the matter-wave solitons and dynamics of Bose-Einstein condensates with time-dependent two- and three-body interactions in time-dependent external potential. We find that when the nonlinear coefficients $g(t)=\left(\sigma_{2} C_{6}{ }^{2} / 2 \rho^{2}\right) e^{-2 \Omega_{1}{ }^{2} t^{2}}, G(t)=\left(\sigma_{3} C_{6}{ }^{2} / 2 \rho^{4}\right) e^{-2 \Omega_{1}{ }^{2} t^{2}}$, the cubic-quintic nonlinear Schrödinger equation supports three families of exact solutions. Moreover, six possible frequencies of harmonic potential are given. Finally, in the case of the harmonic potential $V(x, t)=2 \Omega_{1}\left(1+2 \Omega_{1} t^{2}\right) x^{2}$, we examine the density distributions and dynamics of the matter-wave soliton solutions by analyzing their plots. It is found that 


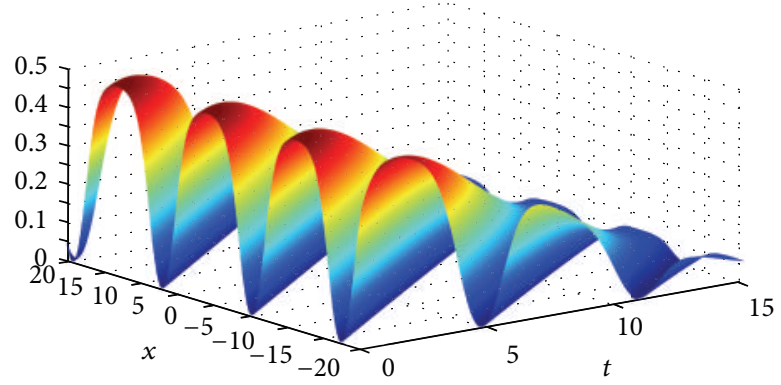

(a) $\left|\psi_{1}(x, t)\right|^{2}$

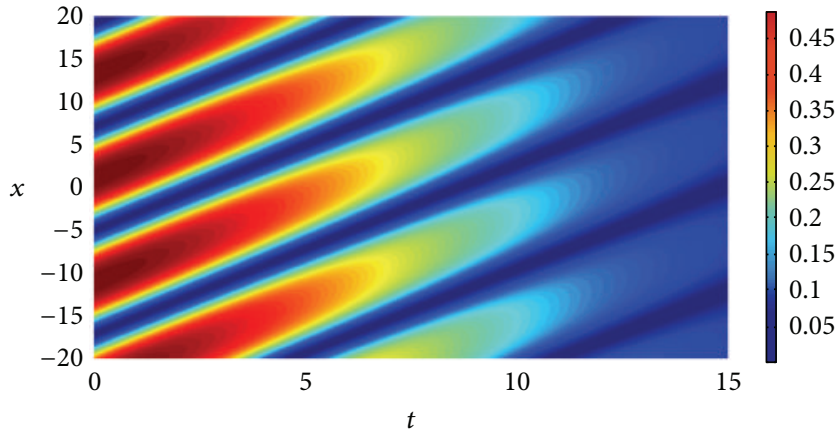

(b) $\left|\psi_{1}(x, t)\right|^{2}$

FIGURE 1: Density distributions of the matter-wave soliton solution $\psi_{1}(x, t)$ : (a) the three-dimensional density distribution and (b) the contour profile.

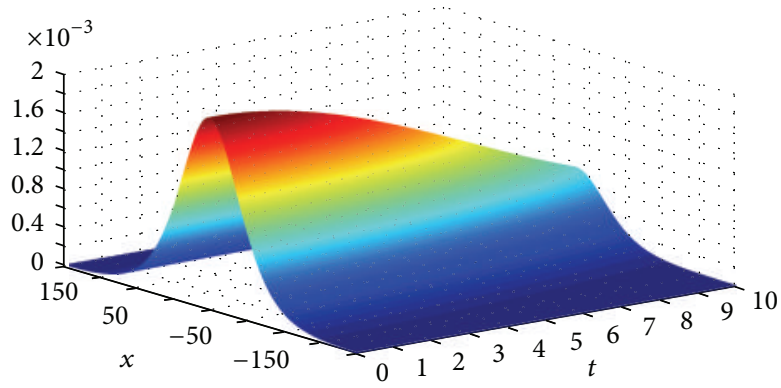

(a) $\left|\psi_{2}(x, t)\right|^{2}$

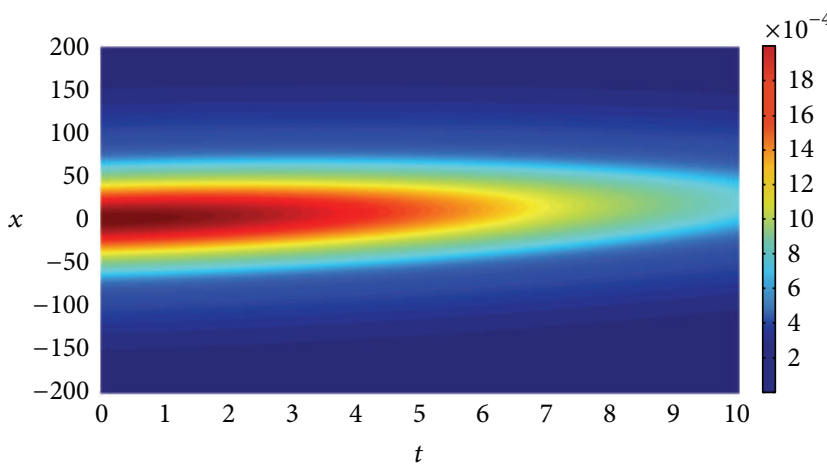

(b) $\left|\psi_{2}(x, t)\right|^{2}$

Figure 2: Density distributions of the matter-wave soliton solution $\psi_{2}(x, t)$ :(a) the three-dimensional density distribution and (b) the contour profile.

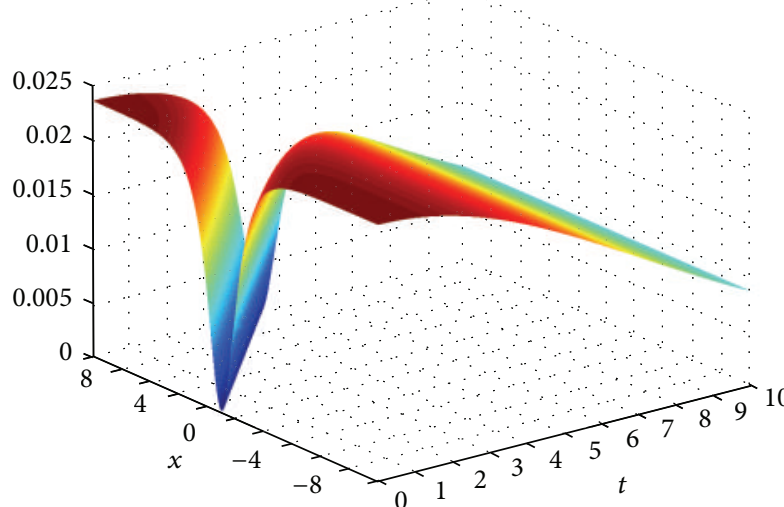

(a) $\left|\psi_{3}(x, t)\right|^{2}$

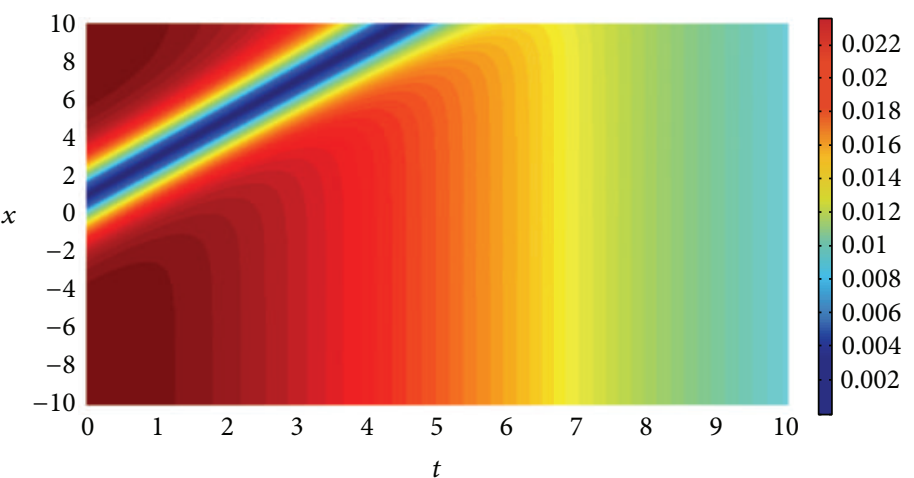

(b) $\left|\psi_{3}(x, t)\right|^{2}$

FIGURE 3: Density distributions of the matter-wave soliton solution $\psi_{3}(x, t)$ : (a) the three dimensional density distribution and (b) the contour profile.

under the effect of time-dependent two- and three-body interactions along with time-dependent harmonic potential, the density distributions of the matter-wave solitons diminish with time. This is consistent with the real Bose-Einstein condensate experiments. There are indeed many papers
[16-18, 21-28] studying the exact solutions of the nonlinear Schrödinger equations by similarity transformations, but they discuss either the matter-wave solitons of Bose-Einstein condensate with two-body interactions or the matterwave solitons of Bose-Einstein condensate with spatially 
inhomogeneous interactions. To our knowledge, the three families of exact solutions (40)-(42) for the cubic-quintic nonlinear Schrödinger are proposed for the first time.

\section{Conflict of Interests}

The authors declare that there is no conflict of interests regarding the publication of this paper.

\section{Acknowledgments}

This work is supported by the Talent Youth Program of Beijing Municipal Commission of Education (Grant no. YETP0984) and the Science and Technology Project of Beijing Municipal Commission of Education (Grant no. KM201311232021).

\section{References}

[1] M. H. Anderson, J. R. Ensher, M. R. Matthews, C. E. Wieman, and E. A. Cornell, "Observation of Bose-Einstein condensation in a dilute atomic vapor," Science, vol. 269, no. 5221, pp. 198-201, 1995.

[2] C. C. Bradley, C. A. Sackett, J. J. Tollett, and R. G. Hulet, "Evidence of Bose-Einstein condensation in an atomic gas with attractive interactions," Physical Review Letters, vol. 75, no. 9, pp. 1687-1690, 1995.

[3] K. B. Davis, M.-O. Mewes, M. R. Andrews et al., "Bose-Einstein condensation in a gas of sodium atoms," Physical Review Letters, vol. 75, no. 22, pp. 3969-3973, 1995.

[4] Y. V. Kartashov, B. A. Malomed, and L. Torner, "Solitons in nonlinear lattices," Reviews of Modern Physics, vol. 83, no. 1, pp. 247-305, 2011.

[5] D. S. Wang, X. F. Zhang, P. Zhang, and W. M. Liu, "Matter-wave solitons of Bose-Einstein condensates in a time-dependent complicated potential," Journal of Physics B: Atomic, Molecular and Optical Physics, vol. 42, no. 24, Article ID 245303, 2009.

[6] D. Wang, S. Song, B. Xiong, and W. M. Liu, "Quantized vortices in a rotating Bose-Einstein condensate with spatiotemporally modulated interaction," Physical Review A-Atomic, Molecular, and Optical Physics, vol. 84, no. 5, Article ID 053607, 2011.

[7] Y. V. Kartashov, V. A. Vysloukh, and L. Torner, "Surface gap solitons," Physical Review Letters, vol. 96, no. 7, Article ID 073901, 2006

[8] R. Yamazaki, S. Taie, S. Sugawa, and Y. Takahashi, "Submicron spatial modulation of an interatomic interaction in a boseeinstein condensate," Physical Review Letters, vol. 105, no. 5, Article ID 050405, 2010.

[9] D. Wang, X. Hu, J. Hu, and W. M. Liu, "Quantized quasi-twodimensional Bose-Einstein condensates with spatially modulated nonlinearity," Physical Review A-Atomic, Molecular, and Optical Physics, vol. 81, no. 2, Article ID 025604, 2010.

[10] J. Belmonte-Beitia, V. M. Pérez-García, V. Vekslerchik, and P. J. Torres, "Lie symmetries and solitons in nonlinear systems with spatially inhomogeneous nonlinearities," Physical Review Letters, vol. 98, no. 6, Article ID 064102, 2007.

[11] Y. Zheng, Y. J. Meng, and Y. W. Liu, "Solitons in Gaussian potential with spatially modulated nonlinearity," Optics Communications, vol. 315, pp. 63-68, 2014.
[12] S. Rajendran, P. Muruganandam, and M. Lakshmanan, "Bright and dark solitons in a quasi-1D Bose-Einstein condensates modelled by 1D GRoss-Pitaevskii equation with timedependent parameters," Physica D: Nonlinear Phenomena, vol. 239, no. 7, pp. 366-386, 2010.

[13] L. P. Pitaevskii, "Vortex lines in an imperfect bose gas," Zhurnal Eksperimental'noi i Teoreticheskoi Fiziki, vol. 40, p. 646, 1961.

[14] C. J. Pethick and H. Smith, Bose-Einstein Condensation in Dilute Gases, Cambridge University Press, Cambridge, Mass, USA, 2008.

[15] A. M. Mateo, V. Delgado, and B. A. Malomed, "Three-dimensional gap solitons in Bose-Einstein condensates supported by one-dimensional optical lattices," Physical Review A-Atomic, Molecular, and Optical Physics, vol. 82, no. 5, Article ID 053606, 2010.

[16] C. P. Search, W. Zhang, and P. Meystre, "Inhibiting three-body recombination in atomic Bose-Einstein condensates," Physical Review Letters, vol. 92, no. 14, 2004.

[17] D. Wang and X. Li, "Localized nonlinear matter waves in a BoseEinstein condensate with spatially inhomogeneous two- and three-body interactions," Journal of Physics B: Atomic, Molecular and Optical Physics, vol. 45, no. 10, Article ID 105301, 2012.

[18] D. Wang, X. Zeng, and Y. Ma, "Exact vortex solitons in a quasi-two-dimensional Bose-Einstein condensate with spatially inhomogeneous cubic-quintic nonlinearity," Physics Letters, Section A: General, Atomic and Solid State Physics, vol. 376, no. 45, pp. 3067-3070, 2012.

[19] M. Abramowitz and I. A. Stegun, Eds., Handbook of Mathematical Functions with Formulas, Graphs, and Mathematical Tables, Dover, New York, NY, USA, 1965.

[20] M. Hazewinkel, Ed., Jacobi Elliptic Functions, Encyclopedia of Mathematics, Springer, New York, NY, USA, 2001.

[21] V. M. Pérez-García, P. J. Torres, and V. V. Konotop, "Similarity transformations for nonlinear Schödinger equations with timedependent coefficients," Physica D: Nonlinear Phenomena, vol. 221, no. 1, pp. 31-36, 2006.

[22] V. N. Serkin, A. Hasegawa, and T. L. Belyaeva, "Nonautonomous solitons in external potentials," Physical Review Letters, vol. 98, no. 7, Article ID 074102, 2007.

[23] J. Belmonte-Beitia, V. M. Pérez-García, V. Vekslerchik, and V. V. Konotop, "Localized nonlinear waves in systems with timeand space-modulated nonlinearities," Physical Review Letters, vol. 100, no. 16, Article ID 164102, 2008.

[24] J. He and H. Li, "Nonautonomous solitary-wave solutions of the generalized nonautonomous cubic-quintic nonlinear Schrödinger equation with time- and space-modulated coefficients," Chinese Physics B, vol. 22, no. 4, Article ID 040310, 2013.

[25] D. Wang, X. Hu, and W. M. Liu, "Localized nonlinear matter waves in two-component Bose-Einstein condensates with time- and space-modulated nonlinearities," Physical Review AAtomic, Molecular, and Optical Physics, vol. 82, no. 2, Article ID 023612, 2010.

[26] D. S. Wang, Y. R. Shi, K. W. Chow, Z. X. Yu, and X. G. Li, "Matterwave solitons in a spin-1 Bose-Einstein condensate with timemodulated external potential and scattering lengths," European Physical Journal D, vol. 67, p. 242, 2013.

[27] D. Wang, S. Yin, Y. Tian, and Y. Liu, "Integrability and bright soliton solutions to the coupled nonlinear Schrödinger 
equation with higher-order effects," Applied Mathematics and Computation, vol. 229, pp. 296-309, 2014.

[28] D. Wang, Y. Ma, and X. Li, "Prolongation structures and matterwave solitons in $F=1$ spinor Bose-einstein condensate with time-dependent atomic scattering lengths in an expulsive harmonic potential," Communications in Nonlinear Science and Numerical Simulation, vol. 19, no. 10, pp. 3556-3569, 2014. 

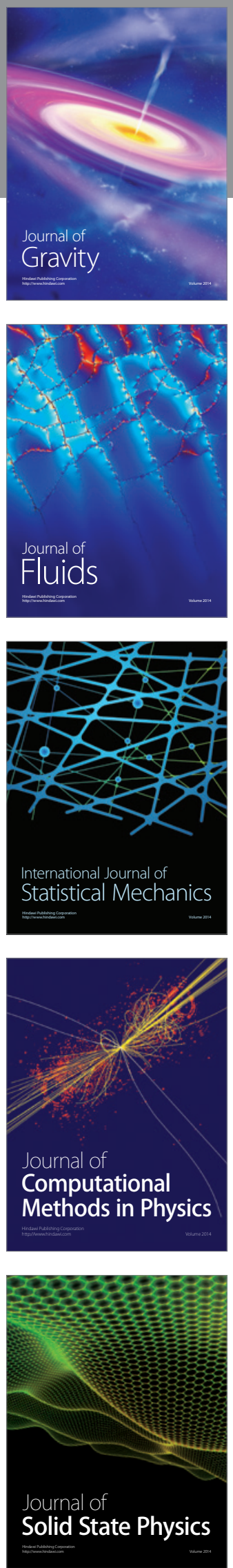

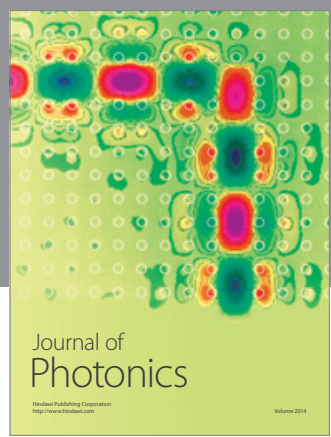

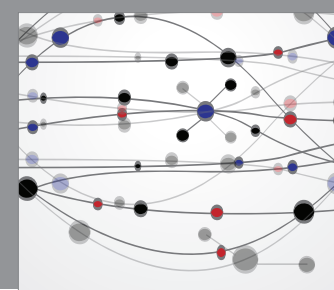

The Scientific World Journal

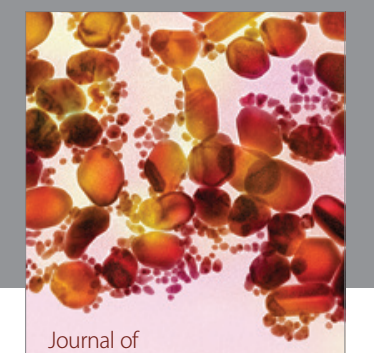

Soft Matter
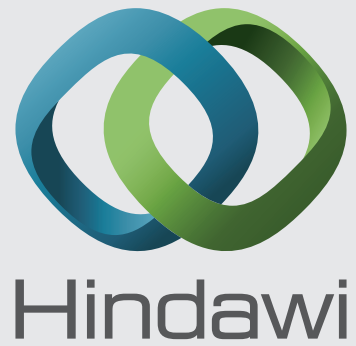

Submit your manuscripts at

http://www.hindawi.com
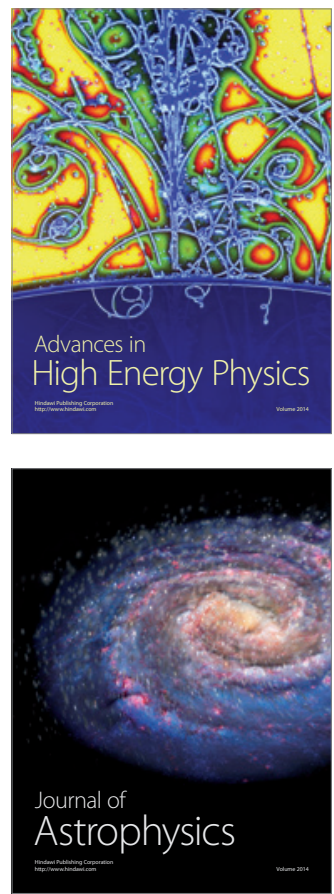
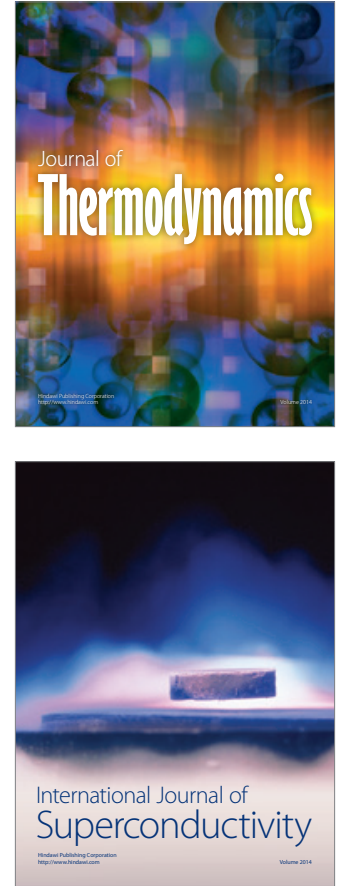
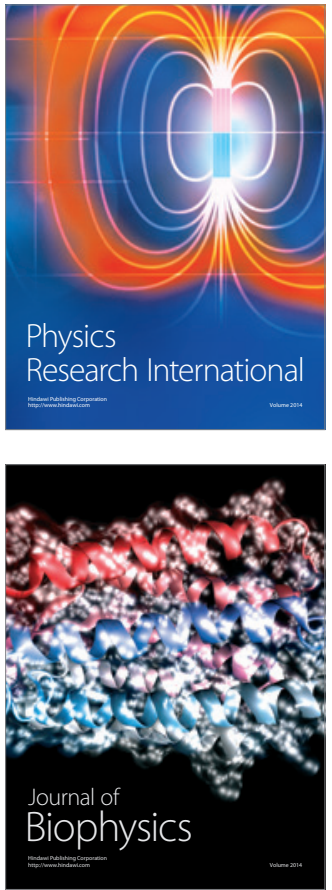
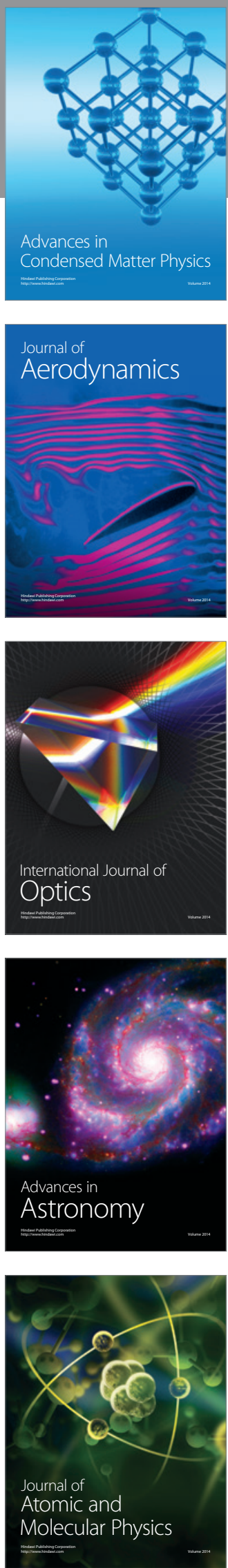